\title{
Memory Effect in the Spatial Series Based on Diamond and Graphite Crystals
}

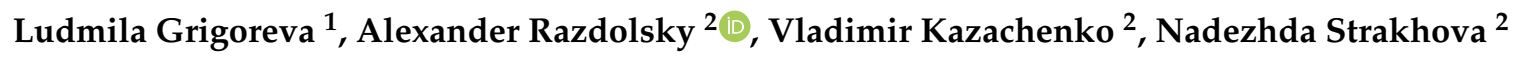 \\ and Veniamin Grigorev ${ }^{2, *(D)}$ \\ 1 Faculty of Fundamental Physical and Chemical Engineering, Lomonosov Moscow State University, \\ Leninskiye Gory 1/51, 119991 Moscow, Russia; ldg@physchem.msu.ru \\ 2 Department of Computer-Aided Molecular Design, Institute of Physiologically Active Compounds of the \\ Russian Academy of Sciences, Severny proezd 1, 142432 Chernogolovka, Russia; rasd@ipac.ac.ru (A.R.); \\ kazach@ipac.ac.ru (V.K.); strakh@ipac.ac.ru (N.S.) \\ * Correspondence: beng@ipac.ac.ru
}

Received: 11 October 2020; Accepted: 16 November 2020; Published: 18 November 2020

\begin{abstract}
To study the relation between the structure of a compound and its properties is one of the fundamental trends in chemistry and materials science. A classic example is the well-known influence of the structures of diamond and graphite on their physicochemical properties, in particular, hardness. However, some other properties of these allotropic modifications of carbon, e.g., fractal properties, are poorly understood. In this work, the spatial series (interatomic distance histograms) calculated using the crystal structures of diamond and graphite are investigated. Hurst exponents $\mathrm{H}$ are estimated using detrended fluctuation analysis and power spectral density. The values of $\mathrm{H}$ are found to be $0.27-0.32$ and $0.37-0.42$ for diamond and graphite, respectively. The calculated data suggest that the spatial series have long memory with a negative correlation between the terms of the series; that is, they are antipersistent.
\end{abstract}

Keywords: diamond; graphite; Hurst exponent; long memory

\section{Introduction}

One of the areas in modern natural science, which attracts the attention of researchers, is fractal geometry and nonlinear dynamics [1-3]. Here, the objects of inquiry can be time or spatial series formed during experimental observations or theoretical calculations. This approach is applied in areas such as astronomy (sun spots) [4], seismology (Earth crust vibrations) [5], physiology (electroencephalograms) [6], meteorology (weather observation data) [7], econophysics (stock quotation) [8], bioinformatics (protein dynamics) [9], and chemistry (concentration changes) [10]. The following two basic problems are solved here: the classification of series and the prediction of their behavior.

To describe dynamic systems quantitatively, researchers have used various quantities to characterize their complexity, ordering, and other properties. One of them is Hurst exponent $\mathrm{H}$, which changes in the range from 0 to 1 and can be used to classify time and spatial series into random and nonrandom series in order to identify a long-range dependence (long memory). In this case, memory is considered in the sense that the future of the process is predicted as a function of its current state. Quantity $\mathrm{H}$ is closely related to fractal geometry, which studies the objects characterized by self-similarity [11]. Here, a scaling region is unlimited only in the case of mathematical objects; in other cases, it is local. Fractal signals, which include time (space) series, are often called long memory processes [12]. They can be classified according to $\mathrm{H}$ into three groups. $\mathrm{H}>0.5$ corresponds to a process with a long-range dependence and a positive correlation. If $\mathrm{H}<0.5$, the process has a 
long-range dependence with a negative correlation. At $\mathrm{H}=0.5$, we observe either a random process or a process with a short-range dependence [13].

Hurst exponents are calculated by various methods, such as the rescaled range (R/S) method [14], detrended fluctuation analysis (DFA) [15], power spectral density (PSD) [16], and fractal wavelet analysis [17]. These methods are based on estimating the exponent of a power function, in which a certain statistic is a dependent variable and a scale (time or spatial) variable is an independent variable. The exponent is fractional in the case of fractal objects. Here, the R/S method is a direct technique for estimating $\mathrm{H}$ and other approaches need recalculation.

A new approach to studying the relation between the structure of a chemical compound and its properties is the use of an interatomic distance histogram as a spatial series [18-20]. In particular, when the homologous series of organic compounds were studied, the series length was found not to affect the Hurst exponent. A simple regression model, which relates $\mathrm{H}$ to the maximum valence bond length, was proposed to explain the differences between homologs.

A classic example of the influence of the structure of a molecule on its properties is represented by the allotropic modifications of carbon, namely diamond and graphite [21]. This group of substances is widely used in practice and has been widely studied. For example, diamond is characterized by an extremely high hardness, which is mainly caused by its crystal structure. Carbon atoms in diamond are in the state of $\mathrm{sp}^{3}$ hybridization. Each atom is located at the center of a tetrahedron and is connected to four other atoms by a high-strength covalent bond. Tetrahedra form a three-dimensional network consisting of layers of six-membered rings. Another modification of carbon is graphite, which has a low hardness. It has a layered structure consisting of six-membered rings, in which every sp ${ }^{2}$-hybridized carbon atom is covalently bonded to three other atoms. Although the physicochemical and biological properties of diamond and graphite have been widely studied, their fractal properties are poorly understood and need further investigation.

The purpose of this work is to calculate and analyze the Hurst exponents of the spatial series based on the interatomic distance histograms of diamond and graphite crystals using DFA and PSD.

\section{Results and Discussion}

The calculated results are shown in Table 1 . The data obtained by the two applied methods are seen to agree with each other. The range of changing $\alpha\left(\mathrm{H}_{\alpha}\right)$ is $0.27 \div 0.40$; that of $-\beta$ is $0.17 \div 0.38$; and $\mathrm{H}_{\beta}, 0.31 \div 0.42$. Diamond crystals have lower values of $\mathrm{H}_{\alpha}$ and $\mathrm{H}_{\beta}$ as compared to graphite crystals. The randomization data $\left(\mathrm{H}_{\alpha}{ }^{\text {rand }}, \mathrm{H}_{\beta}{ }^{\text {rand }}\right)$ correspond to the theoretical expectation $(\mathrm{H}=0.5)$, which confirms the reliability of the calculated results.

Table 1. Parameters $(\alpha, \beta)$, Hurst exponents $(H)$, and standard errors $(\Delta)$, calculated by detrended fluctuation analysis (DFA) $\left(\alpha, \mathrm{H}_{\alpha}, \mathrm{H}_{\alpha}{ }^{\text {rand }}\right)$ иpower spectral density (PSD) $\left(\beta, \mathrm{H}_{\beta}, \mathrm{H}_{\beta}{ }^{\text {rand }}\right)$.

\begin{tabular}{ccccccc}
\hline Crystal & $\boldsymbol{\alpha} \pm \boldsymbol{\Delta}$ & $\mathbf{H}_{\boldsymbol{\alpha}} \pm \boldsymbol{\Delta}$ & $\mathbf{H}_{\boldsymbol{\alpha}}{ }^{\text {rand }} \pm \boldsymbol{\Delta}$ & $-\boldsymbol{\beta} \pm \boldsymbol{\Delta}$ & $\mathbf{H}_{\boldsymbol{\beta}} \pm \boldsymbol{\Delta}$ & $\mathbf{H}_{\boldsymbol{\beta}}{ }^{\text {rand }} \pm \boldsymbol{\Delta}$ \\
\hline D1 & $0.27 \pm 0.01$ & $0.27 \pm 0.01$ & $0.50 \pm 0.02$ & $0.37 \pm 0.03$ & $0.32 \pm 0.02$ & $0.51 \pm 0.02$ \\
D2 & $0.27 \pm 0.01$ & $0.27 \pm 0.01$ & $0.49 \pm 0.02$ & $0.38 \pm 0.03$ & $0.31 \pm 0.02$ & $0.50 \pm 0.01$ \\
G1 & $0.40 \pm 0.01$ & $0.40 \pm 0.01$ & $0.49 \pm 0.02$ & $0.26 \pm 0.03$ & $0.37 \pm 0.01$ & $0.50 \pm 0.01$ \\
G2 & $0.39 \pm 0.01$ & $0.39 \pm 0.01$ & $0.51 \pm 0.02$ & $0.17 \pm 0.03$ & $0.42 \pm 0.01$ & $0.51 \pm 0.01$ \\
\hline
\end{tabular}

In terms of the dichotomic model [12], time and spatial series can be attributed to stationary fractional Gaussian noise ( $\mathrm{fGn})(\alpha<1, \beta<1)$ or nonstationary fractional Brownian motion ( $\mathrm{fBm})(\alpha>1$, $\beta>1$ ), depending on $\alpha$ and $\beta$. These processes are interrelated: integration transforms fGn into fBm and differentiation transforms $\mathrm{fBm}$ into fGn. Since all calculated values fall in the ranges $\alpha<1$ and $\beta<1$, all interatomic distance histograms of the crystals can be attributed to the fGn process.

The Hurst exponent is known to be a quantitative measure of memory for time and spatial series $[13,22,23]$. At $H=0.5$, a series is random and the previous, current, and subsequent values are not related. At $0.5<\mathrm{H}<1$, data exhibit a long-range dependence, which is characterized by a positive 
correlation between the terms of series. An increase in the dependent variable at present is most likely to be retained in the future, and, vice versa, a current decrease is most likely to continue in the future. Such series are called persistent. The range $0<\mathrm{H}<0.5$ is characteristic of long memory with a negative correlation between the terms of series, which is inherent in antipersistent systems. They are characterized by a rapid increase/decrease in the dependent variable in response to a decrease/increase, which leads to oscillations about certain means. As follows from the aforesaid, all spatial series under study are antipersistent (see Table 1).

The interatomic distance histograms under study are statistical fractals characterized by a limited scaling region. In particular, according to DFA data, the scale invariance region of diamond crystals is from $n_{\min }=11$ to $n_{\max }=304$ (Figure 1), which corresponds to distances $r_{\min }=0.11(11 \times 0.01) \AA$ and $r_{\max }=3.04(304 \times 0.01) \AA$. In the case of graphite crystals, we have $n_{\min }=11\left(r_{\min }=0.11 \AA\right)$ иn $n_{\max }=215$ $\left(r_{\max }=2.15 \AA\right)$. At the end of these ranges, an inflection point forms and the angle between $\log (\mathrm{F}(\mathrm{n}))$ and $\log (\mathrm{n})$ increases. However, the new linear segments are short in the scale region and have a small number of points; therefore, it is difficult to estimate their fractal parameters.

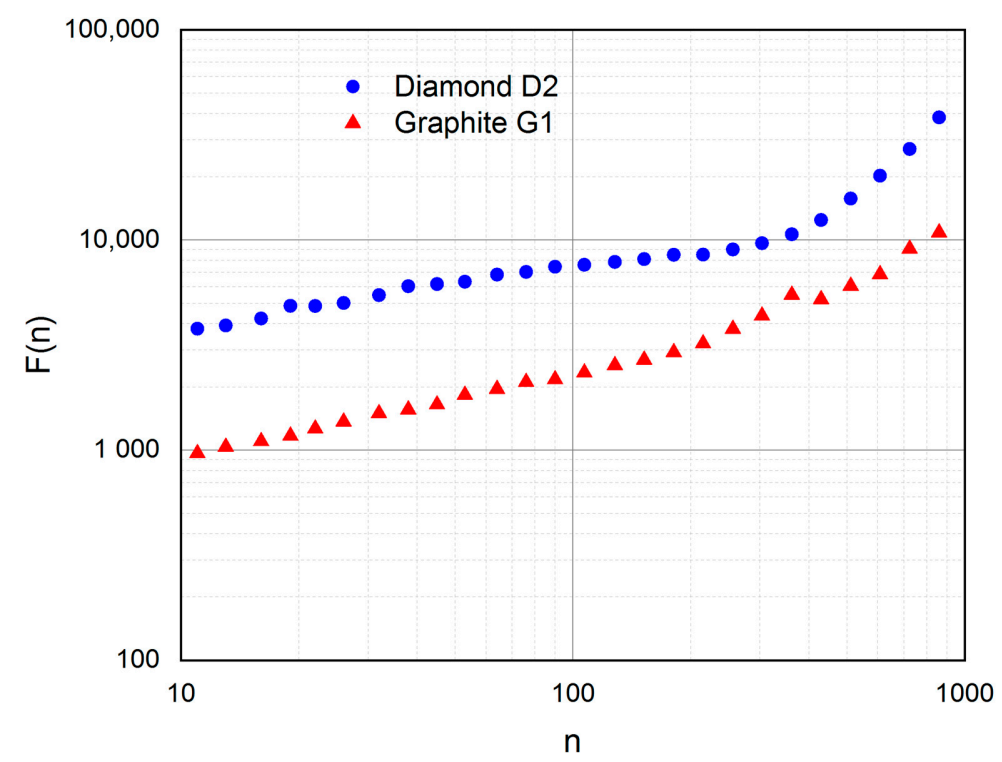

Figure 1. Scale invariance regions of diamond and graphite according to DFA data.

Note that the position of the inflection point in the scaling curve of diamond crystals $(3.04 \div 3.62 \AA)$ approximately corresponds to the lattice parameter $(3.56 \div 3.567 \AA)$ (Table 2$)$. The inflection point characteristics of graphite crystals $(2.15 \div 2.56 \AA)$ agree well with the translation vector a and $b$ lengths $\sim 2.5 \AA$. An increase in the values of $\mathrm{H}$ in the diamond-graphite row $(0.27<0.40)$ is accompanied by a decrease in the average position of the inflection point $(3.33>2.36 \AA)$ and a shortening of the scale invariance region.

One of the fundamental problems in chemistry is the structure-property relationship. In the applied research devoted to this problem, the approach based on the methodology of quantitative structure-activity/property relationship (QSAR/QSPR) is widely used. One of the elements of this approach is to describe the structure of chemical compounds as a matrix of various physicochemical, topological, electronic, and other quantitative characteristics (descriptors). Taking into account the complexity of a molecular structure, we can state that the search for new descriptors with certain information is a challenging problem [24]. Note that, in QSAR/QSPR studies, some descriptors are used to describe the "structure" of chemical compounds (independent variables) and others act as "properties" (dependent variable). Earlier, we proposed a number of descriptors to describe new fractal properties of molecules. They were initially applied to molecules in a gas phase. In addition, the influence of structural changes on the properties and relationship of fractal descriptors with other 
well-known descriptors was investigated. In particular, in $[19,20]$, we calculated and studied the behavior of the Hurst exponents of some organic molecules in a gas phase and found that the series formed using interatomic distance histograms had $\mathrm{H}>0.5$; that is, they are persistent. However, as was found in this work, the spatial series of the two crystalline allotropic modifications of carbon are antipersistent. The resulting discrepancy can be associated with different phase states of the compounds under study (gas or crystal). Further investigations are required to reveal the cause of this discrepancy.

\section{Materials and Methods}

We studied the geometric structures of diamond and graphite crystals. The three-dimensional structures of the substances were constructed with the HyperChem software package [25]. For calculations, we used *.cif files taken from various sources. The lattice parameters and the atomic coordinates in the unit cells were determined using the CrystalMaker software package [26]. The crystal sizes were $343(7 \times 7 \times 7)$ unit cells $(2744$ atoms $)$ for diamond (D1, D2) and 512 unit cells $(8 \times 8 \times 8)(2048$ atoms) for graphite $(\mathrm{G} 1, \mathrm{G} 2)$. Table 2 gives the crystallographic parameters of diamond (cubic system, space group $\mathrm{Fd} 3 \mathrm{~m}$ ) and graphite (hexagonal system, space group $\mathrm{P}_{3} / \mathrm{mmc}$ ).

Table 2. Unit cell parameters of diamond and graphite.

\begin{tabular}{ccccccccc}
\hline Crystal & $\mathbf{a}$ & $\mathbf{b}$ & $\mathbf{c}$ & $\boldsymbol{\alpha}$ & $\boldsymbol{\beta}$ & $\boldsymbol{\gamma}$ & $\mathbf{Z}$ & Source \\
\hline D1 & 3.56 & 3.56 & 3.56 & 90 & 90 & 90 & 8 & {$[25]$} \\
D2 & 3.567 & 3.567 & 3.567 & 90 & 90 & 90 & 8 & {$[27]$} \\
G1 & 2.464 & 2.464 & 6.711 & 90 & 90 & 120 & 4 & {$[27]$} \\
G2 & 2.511 & 2.511 & 6.72 & 90 & 90 & 120 & 4 & {$[28]$} \\
\hline
\end{tabular}

The three-dimensional structures of the crystals were converted into interatomic distance histograms at a step of $0.01 \AA$ (Figure 2). The y-axis is the frequency, i.e., the number of cases in each interval. The obtained spatial series were studied by DFA and PSD.

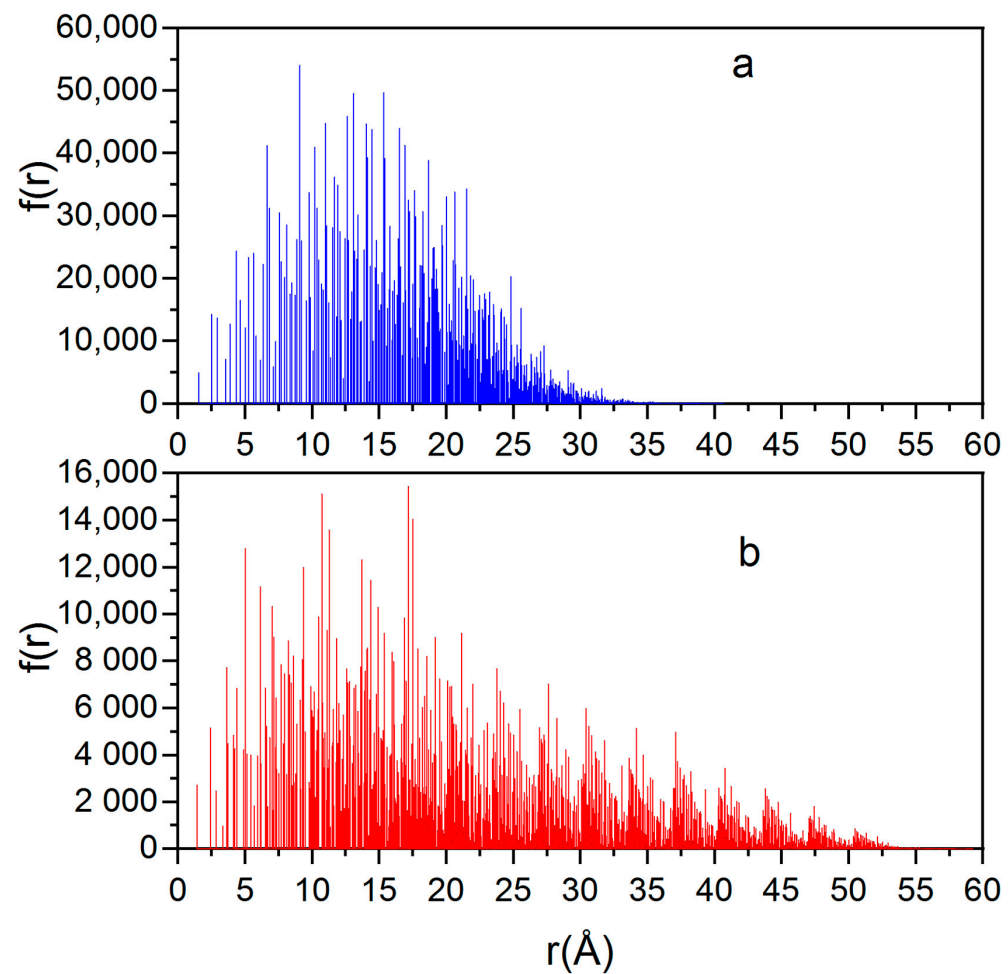

Figure 2. Interatomic distance histograms of (a) diamond D2 and (b) graphite G1. 
The DFA algorithm [15,29] can be represented as the following sequential steps: (1) discrete $X$ series containing $M$ readings was transformed by subtracting the mean $(\mu)$ and summation $\mathrm{y}_{\mathrm{k}}=\Sigma_{\mathrm{i}=1}^{\mathrm{k}}\left(\mathrm{X}_{\mathrm{i}}-\mu\right)$, (2) the obtained series was divided into nonoverlapping blocks of length $\mathrm{n}$, (3) local trend $y_{k, n}$ was estimated in each block using the least squares method, (4) fluctuation function $\mathrm{F}(\mathrm{n})=\left(\left(\Sigma_{\mathrm{k}=1}^{\mathrm{M}}\left(\mathrm{y}_{\mathrm{k}}-\mathrm{y}_{\mathrm{k}, \mathrm{n}}\right)^{2}\right) / \mathrm{M}\right)^{0.5}$ was determined for each block, and (5) exponent $\alpha$ was calculated by double logarithmic transformation of the equation $\mathrm{F}(\mathrm{n})=$ const $\mathrm{n}^{\alpha}$. In this work, we used a linear function to estimate a local trend. The minimum and maximum block sizes were 10 and $M / 4$, respectively. Figure 3 shows an example of the calculation.

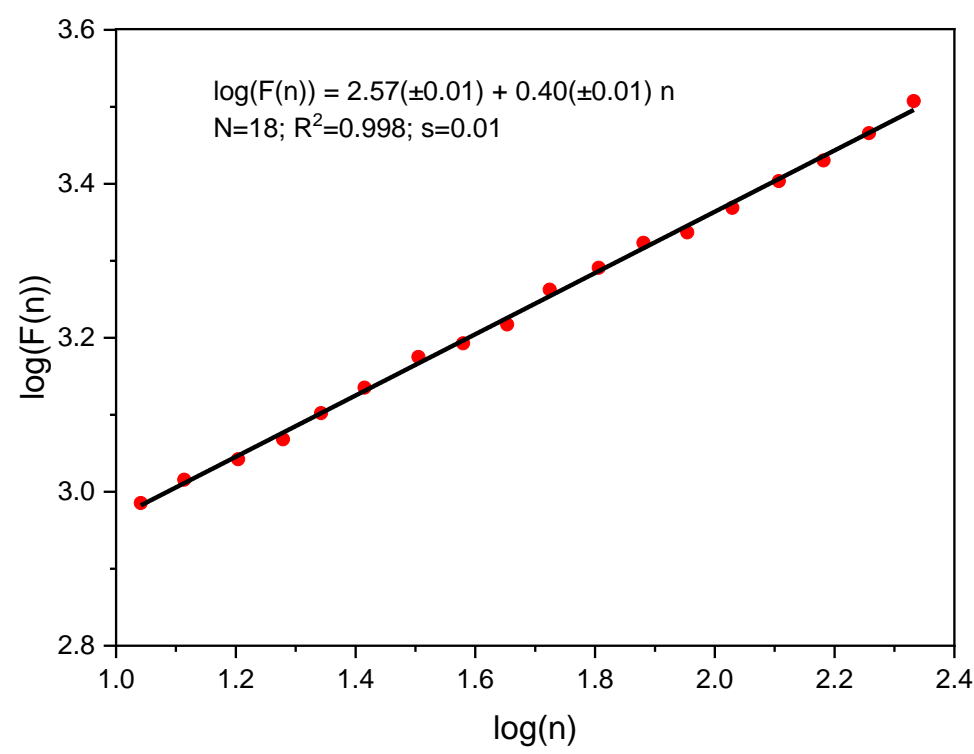

Figure 3. Function F(n) vs. block size $\mathrm{n}$ for graphite G1.

To perform PSD analysis, we used the fast Fourier transform (FFTRF) from the IMSL (International Mathematics and Statistics Library) 7.0 [30]. An initial interatomic distance histogram was converted into the frequency (f) dependence of power density $(\mathrm{P})$. This dependence was subjected to double logarithmic transformation to determine spectral exponent $\beta$. Figure 4 shows an example of the calculation.

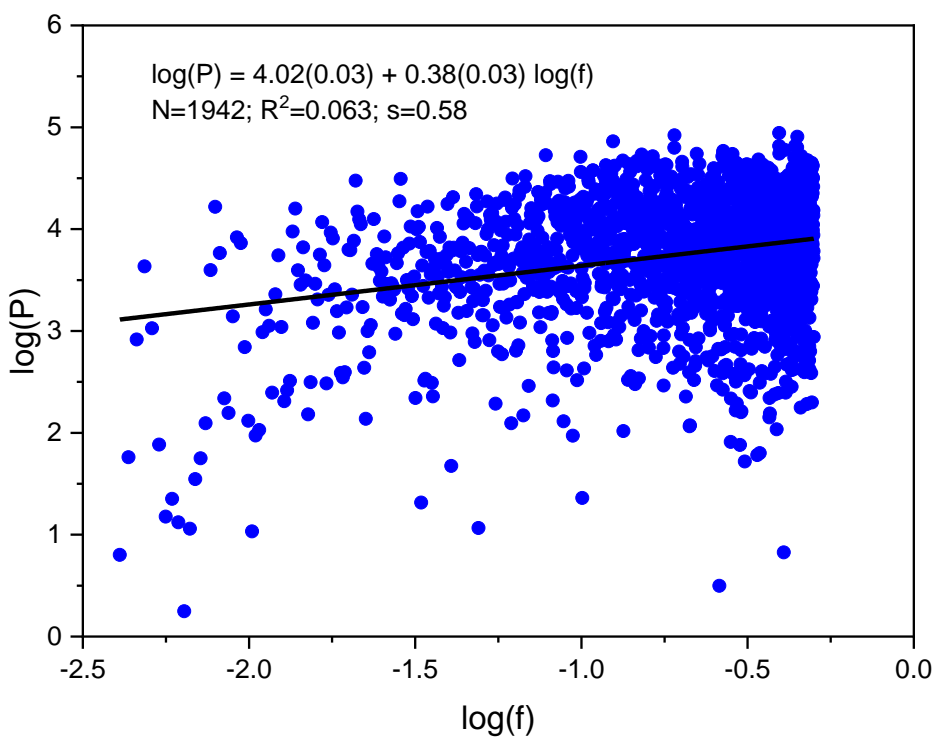

Figure 4. Power spectrum of diamond D2. 
Using the dichotomic model [12] and parameters $\alpha$ and $\beta$, we calculated the Hurst exponents with the formulas $\mathrm{H}_{\alpha}=\alpha(\alpha<1), \mathrm{H}_{\alpha}=\alpha-1(\alpha>1), \mathrm{H}_{\beta}=(\beta+1) / 2(\beta<1)$, and $\mathrm{H}_{\beta}=(\beta-1) / 2(\beta>1)$.

The statistical parameters of regression equations were as follows: $\mathrm{N}$ was the number of points, $\mathrm{R}^{2}$ was the squared coefficient of linear correlation, and $\mathrm{s}$ was the standard deviation.

The Hurst exponents were subjected to internal validation by calculating $\mathrm{H}^{\text {rand }}$ for the randomized series of the initial data (interatomic distance histograms). In this case, the values of $f(r)$ did not change but the order of their appearance in a spatial series changed. To this end, we used 10 random sets of data and averaged the results obtained.

\section{Conclusions}

Using available crystallographic data, we constructed ideal crystal structures of diamond and graphite. Three-dimensional atomic coordinates were used to calculate interatomic distance histograms. The obtained spatial series were analyzed by DFA and PSD. When analyzing Hurst exponents, we found that the series under study had $\mathrm{H}<0.5$; that is, they are antipersistent. When studying scale invariance regions, we revealed inflection points, the position of which correlates with the lattice parameters. The data obtained can be used for the computer-aided molecular design of new substances and materials with allowance for their fractal properties.

Author Contributions: Conceptualization, L.G. and V.G.; methodology, A.R.; software, V.K.; validation, N.S.; formal analysis, V.K.; investigation, L.G. and A.R.; data curation, N.S.; writing-original draft preparation, L.G.; writing-review and editing, V.G.; visualization, V.K. All authors have read and agreed to the published version of the manuscript.

Funding: This research was funded by the Institute of Physiologically Active Compounds, Russian Academy of Sciences, state assignment 2020 no. 0090-2020-0004.

Conflicts of Interest: The authors declare no conflict of interest.

\section{References}

1. Gaite, J. The fractal geometry of the cosmic web and its formation. Adv. Astron. 2019, 6587138. [CrossRef]

2. Grizzi, F.; Castello, A.; Qehajaj, D.; Russo, C.; Lopci, E. The complexity and fractal geometry of nuclear medicine images. Mol. Imaging Biol. 2019, 21, 401-409. [CrossRef] [PubMed]

3. Quaranta, G.; Lacarbonara, W.; Masri, S.F. A review on computational intelligence for identification of nonlinear dynamical systems. Nonlinear Dyn. 2020, 99, 1709-1761. [CrossRef]

4. Covas, E. Spatial-temporal forecasting the sunspot diagram. Astron. Astrophys. 2017, 605, A44. [CrossRef]

5. Ma, K.; Guo, L.; Liu, W. Investigation of the spatial clustering properties of seismic time series: A comparative study from shallow to intermediate-depth earthquakes. Complexity 2018, 7169482. [CrossRef]

6. Roca, J.L.; Rodríguez-Bermúdez, G.; Fernández-Martínez, M. Fractal-based techniques for physiological time series: An updated approach. Open Phys. 2018, 16, 741-750. [CrossRef]

7. Krzyszczak, J.; Baranowski, P.; Zubik, M.; Kazandjiev, V.; Georgieva, V.; Sławiński, C.; Siwek, K.; Kozyra, J.; Nieróbca, A. Multifractal characterization and comparison of meteorological time series from two climatic zones. Theor. Appl. Climatol. 2019, 137, 1811-1824. [CrossRef]

8. Jiang, Z.-Q.; Xie, W.-J.; Zhou, W.-X.; Sornette, D. Multifractal analysis of financial markets: A review. Rep. Prog. Phys. 2019, 82, 125901. [CrossRef]

9. Karain, W.I. Detecting transitions in protein dynamics using a recurrence quantification analysis based bootstrap method. BMC Bioinform. 2017, 18, 525. [CrossRef]

10. Cramer, J.A.; Booksh, K.S. Chaos theory in chemistry and chemometrics: A review. J. Chemom. 2006, 20, 447-454. [CrossRef]

11. Mandelbrot, B. How Long Is the Coast of Britain? Statistical Self-Similarity and Fractional Dimension. Science 1967, 156, 636-638. [CrossRef] [PubMed]

12. Eke, A.; Herman, P.; Kocsis, L.; Kozak, L.R. Fractal characterization of complexity in temporal physiological signals. Physiol. Meas. 2002, 23, R1-R38. [CrossRef] [PubMed]

13. Kristoufek, L. How are rescaled range analyses affected by different memory and distributional properties? A Monte Carlo study. Physica A 2012, 391, 4252-4260. [CrossRef] 
14. Hurst, H. Long term storage capacity of reservoirs. Trans. Am. Soc. Civil. Eng. 1951, 116, 770-799.

15. Peng, C.-K.; Havlin, S.; Stanley, H.E.; Goldberger, A.L. Quantification of scaling exponents and crossover phenomena in nonstationary heartbeat time series. Chaos 1995, 5, 82-87. [CrossRef]

16. Shiomi, K.; Sato, H.; Ohtake, M. Broad-band power-law spectra of well-log data in Japan. Geophys. J. Int. 1997, 130, 57-64. [CrossRef]

17. Simonsen, I.; Hansen, A.; Nes, O.M. Determination of the Hurst exponent by use of wavelet transforms. Phys. Rev. E 1998, 58, 2779-2787. [CrossRef]

18. Grigorev, V.; Raevskii, O. Fractal dimension of the interatomic distance histogram: New 3D descriptor of molecular structure. Russ. J. Gen. Chem. 2011, 81, 449-455. [CrossRef]

19. Grigorev, V.Y.; Grigoreva, L.D. Long-term memory effect in spatial series of model peptides composed of glycine and alanine. Mosc. Univ. Chem. Bull. 2019, 74, 216-222. [CrossRef]

20. Grigorev, V.Y.; Grigoreva, L.D. The study of molecular histograms of interatomic distances in organic homologs on the basis of R/S analysis. Mosc . Univ. Chem. Bull. 2017, 72, 144-148. [CrossRef]

21. Falcao, E.H.L.; Wudl, F. Carbon allotropes: Beyond graphite and diamond. J. Chem. Technol. Biotechnol. 2007, 82, 524-531. [CrossRef]

22. Malamud, B.D.; Turcotte, D.L. Self-affine time series: I. Generation and analyses. Adv. Geophys. 1999, 40, 1-90. [CrossRef]

23. Lopez-Lambrano, A.A.; Fuentes, C.; Lopez-Ramos, A.A.; Mata-Ramirez, J.; Lopez-Lambrano, M. Spatial and temporal Hurst exponent variability of rainfall series based on the climatological distribution in a semiarid region in Mexico. Atmosfera 2018, 31, 199-219. [CrossRef]

24. Muratov, E.N.; Bajorath, J.; Sheridan, R.P.; Tetko, I.V.; Filimonov, D.; Poroikov, V.; Oprea, T.I.; Baskin, I.I.; Varnek, A.; Roitberg, A.; et al. QSAR without borders. Chem. Soc. Rev. 2020, 49, 3525-3564. [CrossRef]

25. HyperChem. Available online: http://www.hyper.com (accessed on 10 October 2020).

26. CrystalMaker. Available online: http://www.crystalmaker.com/index.html (accessed on 10 October 2020).

27. AMCSD. Available online: http://rruff.geo.arizona.edu/AMS/minerals (accessed on 10 October 2020).

28. CCDC. Available online: https://www.ccdc.cam.ac.uk (accessed on 10 October 2020).

29. arXiv. Available online: https://arxiv.org/abs/0804.0747 (accessed on 10 October 2020).

30. IMSL. Available online: https://docs.roguewave.com/en/imsl-main (accessed on 10 October 2020).

Sample Availability: Samples of the compounds D1, D2, G1 and G2 are not available from the authors.

Publisher's Note: MDPI stays neutral with regard to jurisdictional claims in published maps and institutional affiliations.

(C) 2020 by the authors. Licensee MDPI, Basel, Switzerland. This article is an open access article distributed under the terms and conditions of the Creative Commons Attribution (CC BY) license (http://creativecommons.org/licenses/by/4.0/). 